\title{
Um Ambiente Virtual 3D Multiagente com Recomendação Personalizada de Objetos de Aprendizagem
}

\author{
Rodrigo Valença C. Frade ${ }^{1}$, Francisco Milton M. Neto ${ }^{1}$, Rommel W. de Lima ${ }^{1}$, \\ Rodrigo M. de Lima ${ }^{1}$, Luiz Claudio N. da Silva ${ }^{2}$, Rafael C. de Souza ${ }^{3}$ \\ 1Programa de Pós-Graduação em Ciência da Computação - PPGCC \\ Universidade Federal Rural do Semi-Árido - UFERSA \\ Universidade do Estado do Rio Grande do Norte - UERN \\ BR 110 - Km 47 - 59625-900 - Mossoró - RN - Brasil.
}
${ }^{2}$ Superintendência de Tecnologia da Informação e Comunicação - SUTIC
Universidade Federal Rural do Semi-Árido - UFERSA
BR 110 - Km 47 - 59625-900 - Mossoró - RN - Brasil.
${ }^{3}$ Núcleo Tecnológico de Engenharia de Software - NTES
Universidade Federal Rural do Semi-Árido - UFERSA
BR 110 - Km 47 - 59625-900 - Mossoró - RN - Brasil.
\{rodrigo.rvcf, rommel.lima\}@gmail;
\{miltonmendes, rodrigomonteiro, luizclaudio,
rafaelcastro\} dufersa.edu.br

\begin{abstract}
The advancement of technological resources has provided new possibilities in the teaching-learning process. This progress resulted in an improvement to the Distance Education (DE). However, this type of education still faces the serious problem of circumvention that is due mainly to the lack of motivation among students. Thus, the use of new technological trends has been increasingly common with the purpose to provide greater attractiveness for student participation in distance learning courses. Thus, this paper aims to propose a multi-agent virtual environment in three dimensions to support the recommendation of learning objects in order to improve the processes of teaching and learning in $D E$.
\end{abstract}

Resumo. O avanço dos recursos tecnológicos tem proporcionado novas possibilidades nos processos de ensino e aprendizagem. Tal avanço resultou em uma melhoria para a Educação a Distância (EaD). No entanto, esta modalidade de ensino ainda enfrenta o sério problema da evasão que se deve principalmente à falta de motivação por parte dos estudantes. Com isso, a utilização de novas tendências tecnológicas tem sido cada vez mais frequente, tendo como propósito oferecer uma maior atratividade para participação do estudante em cursos a distância. Deste modo, este artigo tem por finalidade propor um ambiente virtual multiagente em três dimensões para apoiar a recomendação de Objetos de Aprendizagem, a fim de melhorar os processos de ensino e aprendizagem na EaD.

\section{Introdução}

A tecnologia tem sido uma grande aliada da Educação a Distância (EaD), tendo em vista que muitos recursos didáticos utilizados atualmente por professores e alunos são 
ferramentas digitais de alto nível de interação que auxiliam, de maneira produtiva e qualitativa, nos processos de ensino e aprendizagem. Atualmente, existem vários desses recursos atrativos, destacando-se os chamados Objetos de Aprendizagem (OA). De acordo com Wiley (2000), um OA consiste em entidades que possibilitam seu uso, reuso ou referência durante a aprendizagem e utilizam tecnologia.

Ainda é possível citar, como referências em avanços tecnológicos, os dispositivos móveis e a comunicação sem fio, pois estes contribuem também de forma considerável para os processos de ensino e aprendizagem. Dentro deste contexto surge outro termo, conhecido como Aprendizagem Móvel (ML - Mobile Learning), que consiste na integração dessas tecnologias para melhorar o processo de aprendizagem [Barbosa 2010].

Os problemas típicos da $\mathrm{EaD}$, tais como a falta de motivação dos estudantes, resultando, muitas vezes, no abandono dos cursos, estimulam atualmente estudos sobre o uso de novas tecnologias [Kermorvant 2010]. A grande vantagem de integrar diferentes tecnologias, de modo que as mesmas interajam entre si para auxiliar nos processos de ensino e aprendizagem, é o fato de todos os usuários poderem usufruir de vários aspectos vantajosos dessa nova abordagem, onde destacam-se a comodidade de prover materiais, acessá-los e visualizá-los, em qualquer horário e lugar [Yau \& Joy 2011].

Atualmente, existe uma grande dificuldade em obter-se a atenção dos jovens para atividades voltadas à aprendizagem. Especificamente na modalidade EaD, este é considerado um grande desafio na presente década. Isso acontece porque os jovens possuem um grande leque de recursos informacionais, bastante interativos, que estão disponíveis ao seu alcance a todo momento. Isso tem atrapalhado o rendimento em atividades de estudo a distância, devido à utilização excessiva desses recursos. Um exemplo são as tecnologias da informação e comunicação (TIC), que dispõem de muito entretenimento, retendo, assim, a atenção dos estudantes.

De acordo com Oliveira e Tedesco (2010), um outro problema está relacionado aos ambientes virtuais de ensino disponíveis no mercado, que, apesar de estarem em grande quantidade, ainda continuam a tratar todos os seus usuários de maneira semelhante. Consequentemente, muitas vezes não há incentivo para uma construção e compartilhamento efetivos do conhecimento, o que também gera problemas de desmotivação e baixos rendimentos. Portanto, se faz necessária a criação de mecanismos que proporcionem aos ambientes, a autonomia de se relacionarem com os conteúdos de aprendizagem segundo o contexto dos alunos e, em seguida, de recomendarem esses, baseando-se nas características de cada um dos alunos.

Tendo em vista essa concepção, o presente artigo propõe o UniVirtual, um sistema que permite simular uma universidade virtual 3D em navegadores de Internet ou nativamente em sistemas Android, onde é possível percorrer, com um personagem estudante, essa universidade virtual que contém alguns Non Playable Characters (NPC) controlados por agentes no intuito de auxiliar nos processos de ensino e aprendizagem através da recomendação de OAs.

Este artigo encontra-se estruturado em sete seções. A Seção 2 mostra uma visão geral de sistemas multiagente. A Seção 3 descreve os objetos de aprendizagem e um de seus padrões. A Seção 4 conceitua sistemas de recomendação. A Seção 5 mostra 
trabalhos já criados com propósitos semelhantes. A Seção 6 descreve a arquitetura do ambiente virtual 3D criado, bem como as tecnologias utilizadas. Por fim, a última seção traz as considerações finais e trabalhos futuros.

\section{Sistema Multiagente}

Russel e Norvig (2003) afirmam que agentes são softwares autônomos que percebem o ambiente por meio de sensores e atuam através de atuadores. Sistemas Multiagente caracterizam-se por um conjunto de agentes colaborativos, a fim de resolver um problema em comum, e que podem resolver um problema maior dividindo o mesmo em vários subproblemas, a fim de otimizar o tempo de solução.

$\mathrm{Na}$ UniVirtual os agentes criados monitoram as atividades do estudante quanto à sua localidade e seu acesso ao sistema. Eles capturam de forma automática as informações dos estudantes e, em seguida, recomendam objetos de aprendizagem que possam ajudar o estudante em determinado assunto ou simplesmente recomendam algo de seu interesse.

\section{Objetos de Aprendizagem}

De acordo com o Comitê de Padronização de Tecnologias de Aprendizagem (LTSC Learning Technology Standard Comitee) do Institute of Electrical and Electronics Engineers (IEEE), um OA consiste em uma entidade material educacional, digital ou não, que pode ser usada para aprendizagem, educação ou treinamento [LTSC 2002].

Silva et al. (2011) alega que a ideia central do conceito de OAs é permitir que os colaboradores educacionais construam objetos educativos relativamente pequenos e que possam ser usados em diferentes contextos de aprendizagem. São conteúdos digitais que permitem alcançar um objetivo educacional promovendo a reusabilidade dos conteúdos utilizados nos processos de ensino e aprendizagem.

Contudo, se faz necessária a garantia dos benefícios propostos na utilização de OAs, deste modo, algumas características são fundamentais e devem ser consideradas na criação de OAs digitais, como, por exemplo: (a) definição da estrutura de navegação; (b) adequação do conteúdo de uma mídia escrita para uma mídia eletrônica; (c) atendimento aos aspectos pedagógicos de ensino; e (d) integração do OA com diferentes tipos de ambientes de EaD [Rodolpho 2009]. A garantia dessas propriedades se dá graças à utilização de padrões de desenvolvimento de OAs.

\subsection{Padrão de Objetos de Aprendizagem SCORM}

Para Kemczinski (2011), os padrões de OAs representam uma forma de organização dos dados para prover comunicação entre diferentes ambientes, bem como o seu acesso e usabilidade, além de garantir sua interoperabilidade. Esses padrões são divididos, de acordo com suas funcionalidades, em: padrões de metadados, de empacotamento, de interface e comunicação e de integração.

Se faz necessário descrever as características do padrão de integração SCORM (Sharabale Content Object Reference Model) utilizado neste trabalho. O SCORM, desenvolvido pela ADL (Advanced Distributed Learning) [ADL 2014], tem como forte característica do padrão de integração, a unificação em um modelo de referência de diferentes tipos de padrões, como, por exemplo, padrões de metadados, empacotamento, 
interface e comunicação, desenvolvidos por outras organizações.

Em outras palavras, conforme da Silva et al. (2012), esse padrão é composto por uma coleção de especificações, adaptadas de múltiplas fontes, que define um conjunto de procedimentos para a construção e o empacotamento de conteúdo instrucional, bem como protocolos para comunicação entre conteúdo e Ambientes Virtuais de Aprendizagem (AVAs). Portanto, a utilização do SCORM possibilita o uso das melhores características de cada um dos padrões disponíveis, o que motivou a sua escolha na abordagem aqui apresentada.

\section{Sistemas de Recomendação}

Silva et al. (2011) descreve que um método de auxílio à escolha de um determinado conteúdo diante de um leque de opções é o uso de Sistemas de Recomendação (SRs). Os SRs auxiliam o usuário no processo de indicação ou recepção de materiais relevantes. Em uma visão geral de um sistema típico de filtragem de informação, as pessoas fornecem recomendações como entradas e o sistema registra e direciona para os indivíduos específicos, ou seja, que são considerados potenciais interessados [Cazella et al. 2012].

Em [Vieira \& Nunes 2012] são apresentados três tipos de SRs: Sistema de Recomendação Baseada em Conteúdo (SRBC), Sistema de Recomendação Colaborativa (SRC) e Sistema de Recomendação Híbrida (SRH). A recomendação baseada em conteúdo é realizada através de informações recuperadas do usuário (perfil, comportamento) e/ou do seu histórico de escolhas. Já a recomendação por filtragem colaborativa leva em consideração escolhas realizadas por usuários com características similares. Por fim, o SRH trata da recomendação através de técnicas utilizadas pelos SRBC e SRC, aumentando as possibilidades e sugerindo conteúdos que não aparentam se relacionar [Martins 2011].

Jácome Júnior et al. (2012) alega que no âmbito dos AVAs, os alunos são expostos a uma grande quantidade de OAs, o que resulta em uma demanda de tempo elevada para realizar escolhas difíceis, tendo em vista que estão ainda em processo de formação e, portanto, sem aptidão suficiente para realizar tais escolhas. Diante desse cenário os SRs podem ser uma boa opção para a indicação de OAs apropriados para estudantes específicos.

\section{Trabalhos Relacionados}

A técnica de recomendação tem sido muito utilizada nos últimos anos em propostas de soluções computacionais. Um exemplo é o trabalho de Silva et al. (2011), que apresenta um ambiente de suporte à aprendizagem móvel capaz de realizar a recomendação sensível ao contexto de OAs, definidos em um formato padrão, através do uso de ontologias e de um sistema multiagente. Este ambiente considera perfis de estudantes, localização dos mesmos, e escolhas similares de OAs.

Em [Yau \& Joy 2011], é apresentada uma arquitetura de aprendizagem móvel sensível ao contexto composta por três componentes principais: o perfil do estudante, um mecanismo de personalização e um repositório de OAs. O perfil do estudante serve para armazenar suas preferências de aprendizagem móvel, capturadas através de um questionário respondido pelo estudante. $\mathrm{O}$ mecanismo recebe esse perfil e combina as 
informações dele com aquelas obtidas dinamicamente através de sua interação com o ambiente. Seguidamente, são comparadas todas as informações obtidas com os metadados dos OAs disponíveis no repositório. O sistema então recomenda OAs adequados ao estudante com base nas características do seu contexto.

Já Wiedemann et al. (2013) propõem um modelo para recomendação de OAs baseado na análise de similaridade entre históricos de consultas realizadas em repositórios. O modelo recebe a sequência de OAs consultados durante a sessão atual do usuário e localiza sessões cujas sequências de OAs consultados sejam similares à sequência da sessão corrente. Em seguida, os OAs encontrados nas sessões similares são então recomendados.

Para o desenvolvimento da UniVirtual foram reunidas as principais características dos trabalhos citados anteriormente. Entretanto, o que difere o presente trabalho dos demais é o desenvolvimento de um ambiente virtual 3D integrado a todas essas técnicas, possibilitando a recomendação autônoma e personalizada de OAs baseada no contexto que os estudantes se encontram em um dado momento com o auxílio de agentes de software.

\section{Sistema 3D Multiagente para Recomendação de Objetos de Aprendizagem}

$\mathrm{Na}$ arquitetura proposta neste artigo, os estudantes precisam, inicialmente, estarem cadastrados no LMS Moodle. Neste cadastro são fornecidas informações bastante relevantes que caracterizam o seu perfil e que servirão futuramente para a criação de um determinado contexto. Apenas após essa etapa é possível se autenticar à UniVirtual.

A comunicação com a base de dados do Moodle no ato da autenticação é efetuada a partir de scripts executados pela UniVirtual. Já a atuação dos agentes funciona através de um sistema multiagente acionado por mensagens, e que necessita dessa mesma base para acessar os metadados do estudante. Esse quadro básico de comunicação inicial entre os componentes encontra-se esboçado na Figura 1.

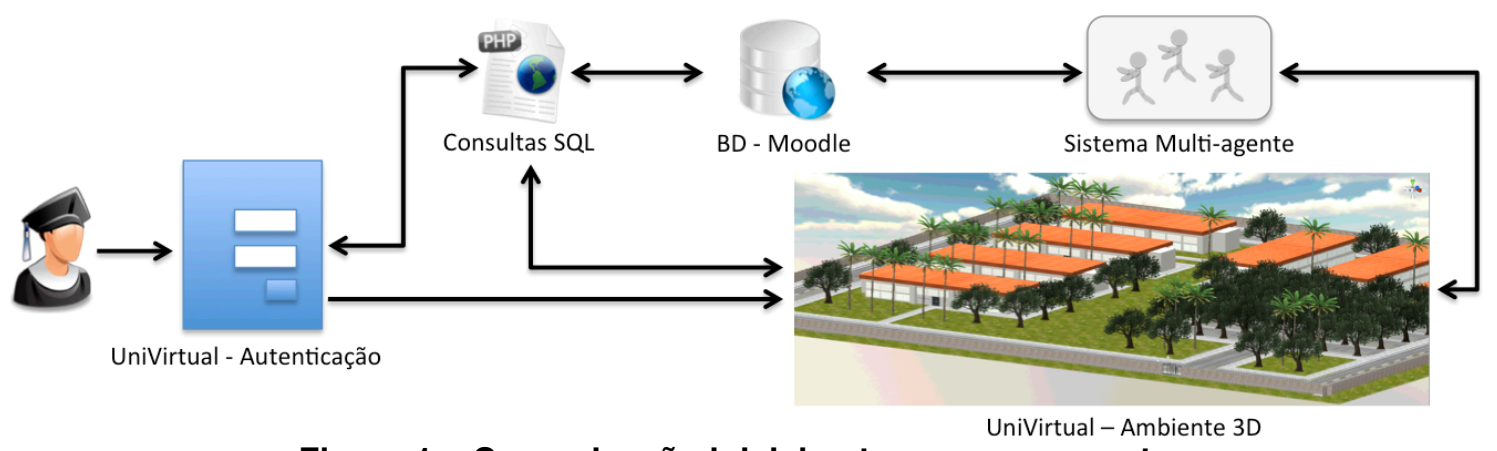

Figura 1 - Comunicação inicial entre os componentes

Após a autenticação é carregado o cenário da universidade virtual, que contempla sete departamentos de diversas áreas de estudo. Esse ambiente tridimensional pode ser explorado através de um personagem que representa o estudante. Cada departamento possui um professor virtual que interage com o usuário através da oferta de OAs de forma autônoma. O que caracteriza essa autonomia é a possibilidade de identificação da proximidade do estudante por meio de uma implementação básica de Inteligência Artificial (IA) associada ao sistema multiagente que encontra-se integrado 
ao ambiente e que trata, por sua vez, da recomendação dos OAs baseada na localidade e perfil do estudante em questão.

É possível se ter uma visão geral dos componentes do sistema através da arquitetura ilustrada na Figura 2.

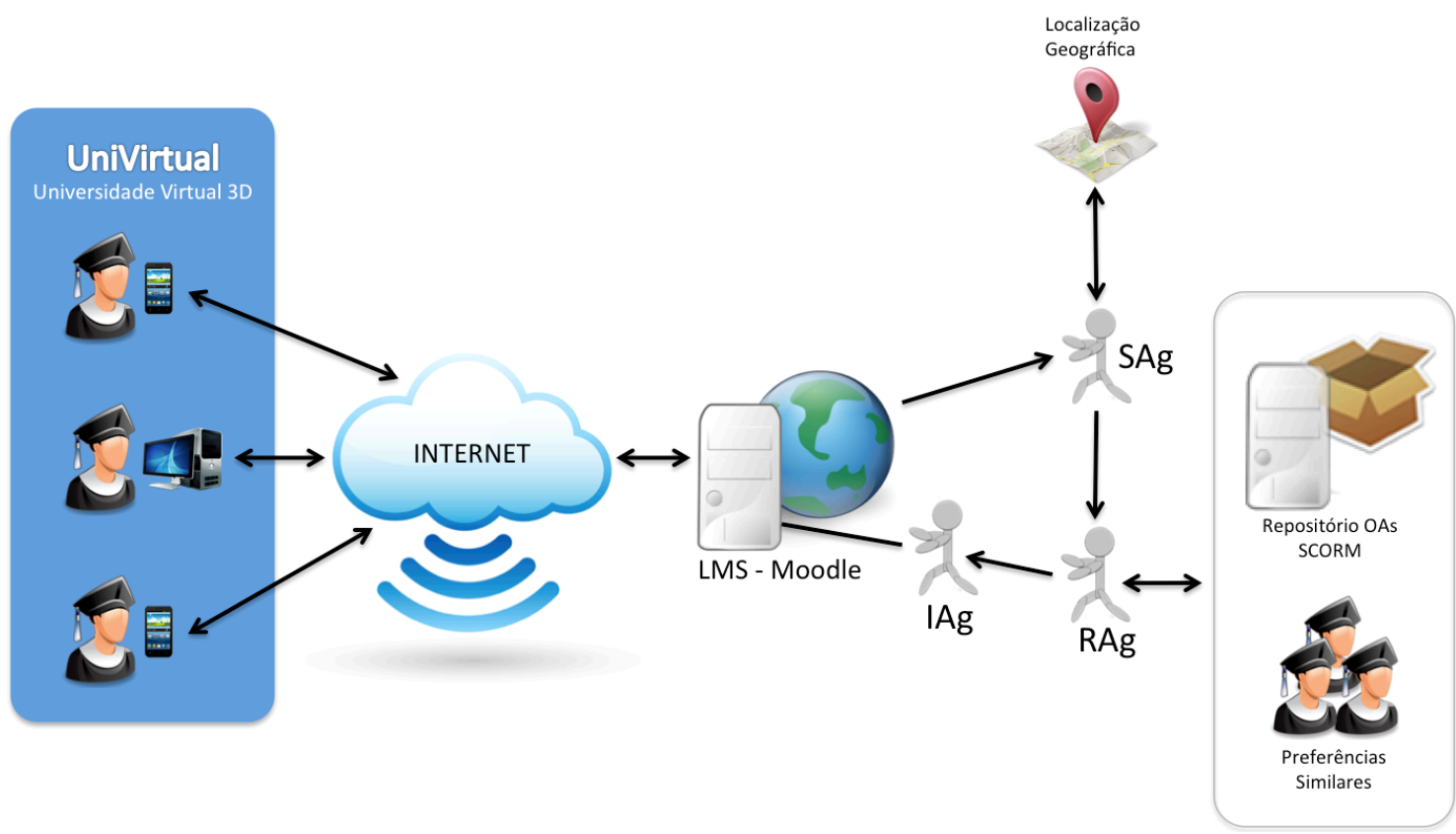

Figura 2. Arquitetura do ambiente proposto

$\mathrm{O}$ ambiente 3D descrito nesta abordagem foi desenvolvido utilizando a engine Unity 3D, uma ferramenta específica para a criação de jogos $2 \mathrm{D}, 3 \mathrm{D}$ e conteúdo interativo [Unity 2014]. Alguns objetos, tais como: prédios, cadeiras e computadores, foram fornecidos através de uma parceria com os membros do projeto UFERSA Virtual [Souza et al. 2013]. Para o desenvolvimento dos agentes foi utilizado o JADE (Java Agent Development Framework), uma plataforma específica para o desenvolvimento e execução de sistemas multiagente [JADE 2014].

Um outro componente imprescindível no auxílio ao mecanismo de recomendação é o repositório de OAs do Moodle. Porém, tem-se como pré-requisito a padronização dos OAs conforme o SCORM. Por meio dos metadados descritos nesse padrão o agente terá autonomia de comparar as informações dos OAs com os perfis dos estudantes/usuários e realizar a recomendação de conteúdos de maneira adequada.

No sistema de recomendação proposto estão sendo considerados dois tipos de recomendação: (i) Recomendação Colaborativa, que compara estudantes/usuários com preferências semelhantes e (ii) Recomendação Baseada em Conteúdo, que compara o conteúdo dos OAs em relação aos perfis dos estudantes. Assim, se tem um Sistema de Recomendação Híbrida (SRH).

\subsection{Atuação dos Agentes de Software}

Na proposta aqui abordada foi necessária a implementação de três tipos de agentes, como é possível perceber na Figura 1: Agente Estudante (SAg - Student Agent), Agente Recomendador (RAg - Recommender Agent) e Agente de Interface (IA - Interface Agent). 
A responsabilidade dos SAgs consiste no monitoramento das atividades dos estudantes e recuperação das suas preferências de aprendizagem, obtidas através dos seus perfis de usuários, incluindo os seus históricos em opções de OAs. O seu comportamento é realizado a partir da autenticação do estudante na UniVirtual e consiste em, baseado nos históricos de escolhas, identificar outros estudantes com históricos contendo escolhas similares. Estes agentes também são capazes de capturar as informações do contexto dinâmico do estudante, além de sua localização geográfica. Por fim, todas essas informações são enviadas para o RAg.

Por sua vez o RAg, de acordo com as informações fornecidas pelos SAgs e as obtidas dos OAs disponíveis no repositório, detecta os OAs que correspondam ao contexto atual do estudante. Inicialmente ele encontra o OA mais adequado de acordo com a Recomendação Baseada em Conteúdo e, em seguida, com base nas informações já obtidas, considera quais desses OAs foram aceitos também por estudantes que possuem perfis similares ao estudante em atividade, caracterizando a Recomendação Colaborativa. Com isso, é possível obter-se uma taxa de acerto considerável no momento da recomendação. Depois de selecionar os OAs adequados, o RAg repassa estes para o IAg que, por sua vez, irá executar a oferta ao estudante.

Na Figura 3 encontram-se o personagem que representa o estudante autenticado prestes a entrar no departamento de matemática e, em seguida, o momento em que ocorre a recomendação de OAs.
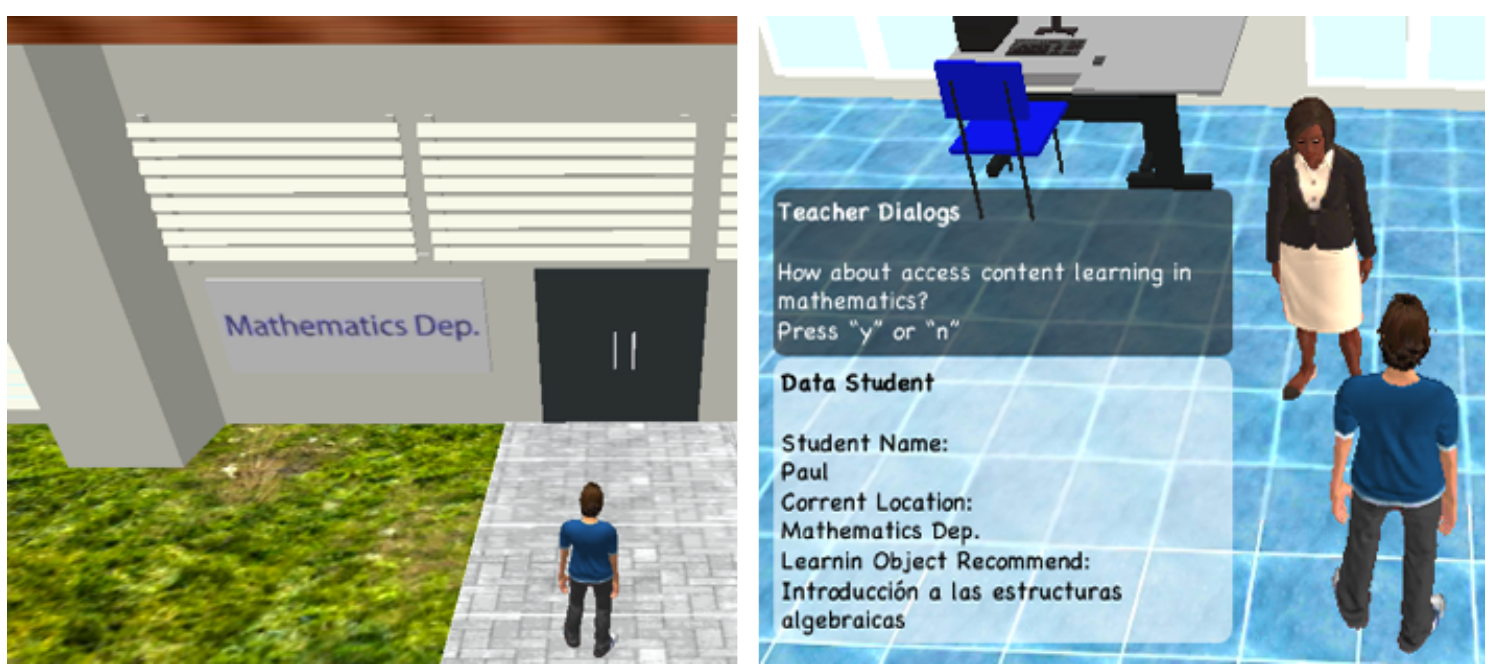

Figura 3. Cenário inicial e sugestão de OAs

O papel principal do IAg é avaliar a relevância do OA a ser recomendado com base no contexto do estudante e, caso o OA não seja o mais adequado, rejeitar a recomendação. Outra responsabilidade do IAg é informar ao professor (ou tutor) o conteúdo que foi sugerido. Para se ter um melhor entendimento sobre toda a interação entre os Agentes, a Figura 4 ilustra um diagrama de sequência que apresenta a comunicação entre eles. 


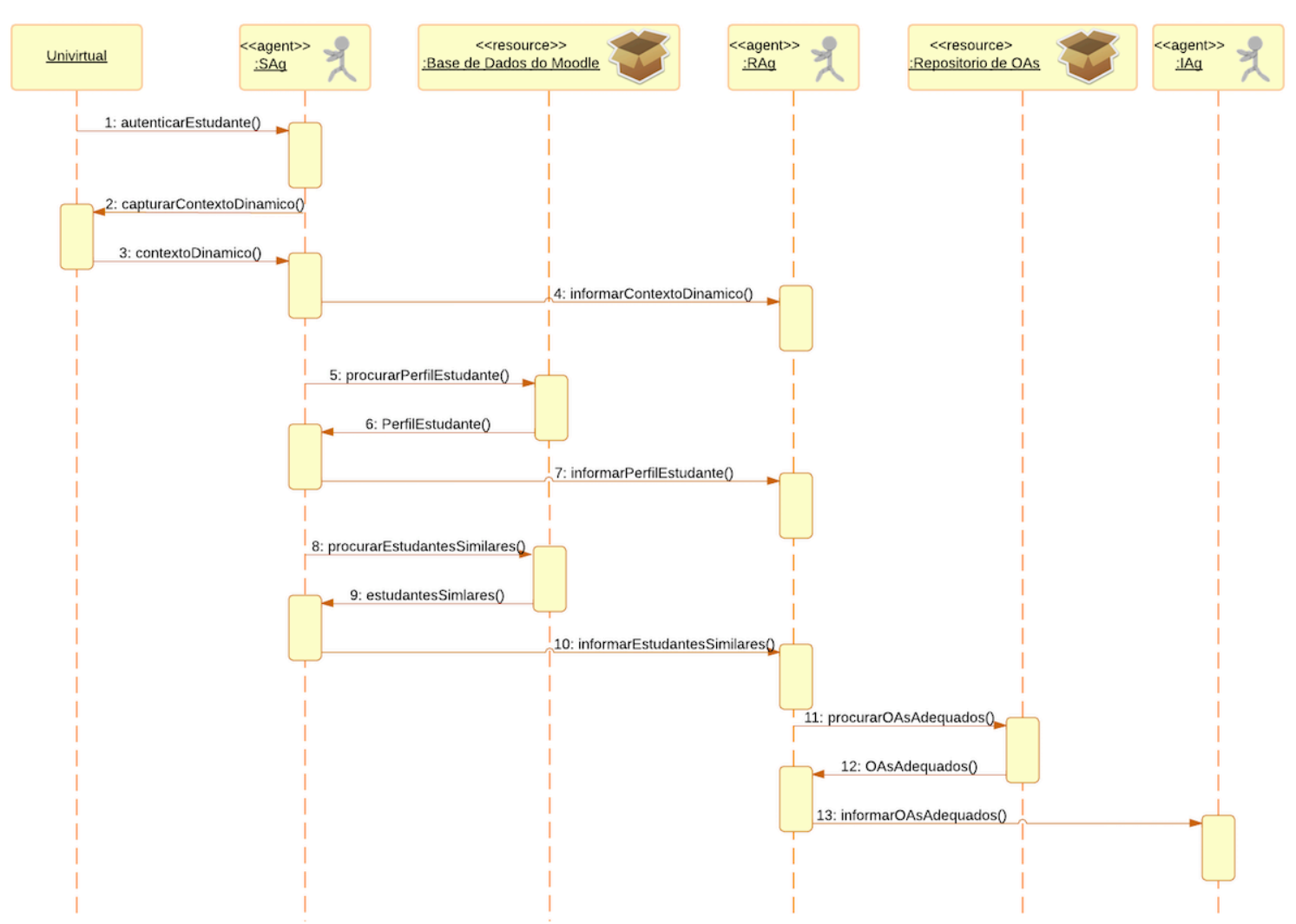

Figura 4. Interação dos Agentes

\subsubsection{Recomendação Baseada em Conteúdo}

O mecanismo de recomendação baseada em conteúdo utilizado considera as informações de local e horário preferidos de estudo e área de interesse do estudante. Essas informações são ponderadas de acordo com o nível de influência que cada uma exerce no modelo de aprendizagem do estudante. Assim, a forma de identificar os OAs adequados ao contexto do estudante na UniVirtual é realizada de acordo com a Equação 1 .

$$
F R=((A I * 5)+(L P * 3)+(H P * 2)) / 10-F A
$$

Deste modo, o Fator de Recomendação (FR), determinado pelo RAg, é influenciado, principalmente, pela Área de Interesse (AI) do estudante, tendo, portanto, peso 5. O Local Preferido (LP), por apresentar uma grande influência na capacidade de concentração do estudante, recebe peso 3. Por fim, o Horário Preferido (HP) de estudo também é de interesse pelo fato de poder exercer influência no nível de concentração e, consequentemente, influenciar positiva ou negativamente a recomendação de um OA. Já o Fator de Ajuste (FA) diz respeito a um fator que pode ser estabelecido pelo professor a fim de aumentar (quando o FA for menor) ou diminuir (FA maior) o impacto que o contexto do usuário exerce para a recomendação de OAs. Os valores numéricos de AI, LP e HP são obtidos com base nos valores capturados dinamicamente e naqueles previamente definidos de contexto estático dos estudantes.

Para definir, de forma dinâmica, o valor que representa o quão adequado e determinado o OA é em relação à área de interesse de um estudante, são consideradas três características do OA: descrição, título e palavras-chave. O RAg, por sua vez, verifica a incidência de palavras de interesse do estudante nessas três características do 
OA. Em seguida, esses valores são ponderados pelo RAg conforme a Equação 2.

$$
A I=((P C * 3)+(D * 2)+(T * 1)) / 6
$$

A Equação 2 mostra que o maior peso é dado às Palavras-Chaves (PC), visto que representam melhor os assuntos tratados no OA. A Descrição (D) do OA nos fornece uma visão geral de como os seus diversos assuntos estão integrados. Por fim, o Título (T) representa uma influência menor, dentre as três características, por não conter uma gama de palavras relacionadas ao OA tão abrangente quanto as palavras-chaves.

Para a definição dinâmica do fator LP ao utilizar a UniVirtual através de um dispositivo móvel, foi implementada uma classe responsável por capturar a localização geográfica do estudante a partir de uma API (Aplication Programming Interface) integrada ao GPS (Global Positioning System) desse dispositivo, tornando possível obter a sua latitude e longitude. Essa informação é, então, enviada pela aplicação cliente do dispositivo móvel ao SAg, e este, por sua vez, realiza a geocodificação reversa ${ }^{1}$ das informações antes de enviá-las ao RAg.

A captura dinâmica do horário atual é feita pelo SAg no momento de autenticação do estudante no ambiente UniVirtual. Essa informação servirá para que o RAg defina o valor numérico atribuído ao fator HP.

\section{Considerações Finais e Trabalhos Futuros}

Foi descrito neste artigo, a implementação de uma arquitetura baseada em agentes para recomendação de OAs em um ambiente virtual 3D. A solução proposta objetiva tornar a aprendizagem virtual personalizada e adequada dependendo das necessidades de cada estudante. Essa arquitetura pode ser aplicada em qualquer ferramenta de gestão de aprendizagem.

Como trabalhos futuros, pretende-se testar o ambiente em um curso de ensino a distância, a fim de validar o sistema e torná-lo disponível para todos. Espera-se também utilizar a teoria da carga cognitiva [Santos et al. 2009] com o objetivo de balancear a carga de recomendação de conteúdos de forma personalizada para cada estudante.

Também objetiva-se a implementação de uma função multiplayer ao ambiente 3D, de modo a permitir uma interação entre vários estudantes, inclusive de instituições distintas na UniVirtual em um mesmo instante, de modo a tornar a ferramenta cada vez mais atrativa e estimulante para os estudantes.

\section{Referências}

ADL (2014) “Advanced distributed learning”, Disponível em: http://www.adlnet.org. Acesso em: 10 mai. 2014.

Barbosa, A. S. E. S. J. (2010). M-Learning e U-Learning: Novas Perspectivas da Aprendizagem Movel e Ubiqua. [S.1.]: Pearson.

Cazella, S. C.; Bhear, P.; Schneider, D.; Silva, K. K.; Freitas, R. (2012) “Desenvolvendo um Sistema de Recomendação de Objetos de Aprendizagem baseado em Competências para a Educação: relato de experiências ". In Anais do XXIII Simpósio Brasileiro de Informática na Educação, SBIE, Rio de Janeiro, RJ.

\footnotetext{
${ }^{1}$ Conversão de latitude e longitude em endereço, ou seja, rua, número, bairro, cidade, estado ou país.
} 
da Silva, T. R., Lima, R. W., Mesquita, H. H., Marques, C. K., Costa, R. D., Rocha, S. M., \& Medeiros, R. A. (2012). OBA-MC: um modelo de Objeto de Aprendizagem centrado nos processos de ensino e aprendizagem utilizando o padrão SCORM. In Anais do Simpósio Brasileiro de Informática na Educação (Vol. 23, No. 1).

JADE (2014) "Java development framework: an open-source platform for peer-to-peer agent based applications". Disponível em: http://jade.tilab.com/. Acesso em: 23 jun. 2014.

Jácome Júnior, L., Neto, F. M. M., Flores, C. D., da Silva, L. C. N., Sombra, E. L., \& da Costa, A. A. L. (2012). Uma Extensão do Moodle para Recomendação Ubíqua de Objetos de Aprendizagem. Renote, $10(3)$.

Kemczinski, A., Hounsell, M. D. S., Gasparini, I., Gehrke Filho, R., \& da Silva, T. C. (2011). Repositório de Objetos de Aprendizagem para a Área de Computação e Informática-ROAI. In Anais do Simpósio Brasileiro de Informática na Educação (Vol. 1, No. 1).

Kermorvant, A. Y. J.-M. L. F. (2010). Segae: A serious game authoring environment. 10th IEEE International Conference on Advanced Learning Technologies. Disponível em: http://dx.doi.org/10.1109/ICALT.2010.153. Acesso em: 05 nov. 2013.

LTSC (2002) “Draft Standard for Learning Object Metadata. IEEE Standard 1484.12.1”. LTSC, Learning Technology Standards Committee, New York: Institute of Electrical and Electronics Engineers. Disponível em: http://ltsc.ieee.org/wg12/files /LOM_1484_12_1_v1_Final_Draft.pdf. Acesso em: 07 dez. 2013.

Martins, H. N. J., Costa, E. B., Oliveira, T. T. M., Bittencourt, I. I. (2011). Sistema de Recomendação Híbrido para Bibliotecas Digitais que Suportam o Protocolo OAI-PMH, Artigo Completo, In: XXII Simpósio Brasileiro de Informática na Educação, SBIE, Aracaju, SE.

Oliveira, E. A.; Tedesco, P. (2010). i-collaboration: Um modelo de colaboração inteligente personalizada para ambientes de ead. Revista Brasileira de Informática na Educação, v. 18, n. 01, p. 17.

Rodolpho, E. R. (2009) “Convergência digital de objetos de aprendizagem SCORM”, Dissertação de Mestrado, Universidade Estadual Paulista Júlio de Mesquita Filho -UNESP, São José do Rio Preto, SP.

Russell, R. \& Norvig, P. (2003) “Artificial Intelligence, A Modern Approach 2nd Edition”, Prentice Hall.

Santos, L. M. A., Reategui, E., Tarouco, L. (2009) "A Inserção de um Agente Conversacional Animado em um Ambiente Virtual de Aprendizagem a partir da Teoria da Carga Cognitiva”, Artigo Resumido, In Anais do XX Simpósio Brasileiro de Informática na Educação, SBIE, Florianópolis, SC.

Silva, N. C. L., Mendes Neto, F. M. e Júnior J. L. (2011) "MobiLE: Um Ambiente Multiagente de Aprendizagem Móvel para Apoiar a Recomendação Sensível ao Contexto de Objetos de Aprendizagem", Artigo completo, In Anais do XXII SBIE - XVII WIE, Aracaju, SE, 21-25 de Novembro de 2011.

Souza, D. F. L. ; Castro, A. F. ; Maia, H. W. (2013). UFERSA Virtual - Campus virtual inteligente para navegação e integração de serviços acadêmicos. In XIX Seminário de Iniciação Científica - SEMIC.

Unity (2014) "Development engine for the creation of 2D and 3D games and interactive content". Disponível em: http://unity3d.com/. Acesso em: 20 mai. 2014.

Vieira, F. J. R. and Nunes, M. A. S. N. (2012). DICA: Sistema de Recomendação de Objetos de Aprendizagem Baseado em Conteúdo, Scientia Plena, v. 8.

Wiedemann, T., Barbosa, J. L., \& Rigo, S. J. (2013). Um Modelo para Recomendação de Objetos de Aprendizagem Baseado em Similaridade de Sessões. In Anais do Simpósio Brasileiro de Informática na Educação (Vol. 24, No. 1).

Wiley, D. A. (2000). Connecting learning objects to instructional design theory: A definition, a metaphor, and a taxonomy. In D. A. Wiley (Ed.), The Instructional Use of Learning Objects. Disponvel em: http://reusability.org/read/ chapters/wiley.doc. Acesso em: 24 fev. 2014.

Yau, J. Y. K., \& Joy, M. (2011). A context-aware personalised m-learning application based on mlearning preferences. International Journal of Mobile Learning and Organisation, 5(1), 1-14. 